Trieste

\title{
STORIA DI PAROLE ED ETIMI TRIESTINI
}

\author{
Petes "grappa, acquavite"
}

0. Dopo quanto esaurientemente esposto da M. Doria in GDDT e GDDT Suppl. sussistono ancora - a mio modesto parere - elementi validi per riscrivere, almeno parzialmente, la storia della parola "caratterizzante" triestina petès "bevanda superalcoolica (soprattutto di pessima qualità)", "grappa, acquavite" e simili, anche se questi stessi elementi non si appalesino, purtroppo, decisivi - come vedremo - per fissare definitivamente l'etimo della medesima. Vale, comunque, sempre la pena di ripercorrerne la documentazione, al fine di recuperare almeno una parte della "storia" della parola in questione.

Seguiamo, quindi, passo per passo le tappe di questo nostro nuovo approccio:

1. Impiego di petes nel triestino. Non vi recuperiamo nulla di sorprendentemente nuovo. Si può, al massimo, tener presente la variante "arcaica" petesse (1851), forse utilizzabile per il discorso che faremo succesivamente. Formalmente essa ha l'area di una forzata italianizzazione a partire da petes, quasi alla pari del petesso fiumano (già citato del GDDT). La spinta potrebbe essere stata data da coppie alternanti preesistenti nel triestino tipo cales/calesse "calesse" o anche pes/pesse "pesce". Tra i derivati già noti una postilla possiamo fare a petessaría "liquoreria", che si è formata nel nostro dialetto ben prima del neologismo italiano grapperia (cfr. G. Pittana "Passaparola" sett. 1987 p. 196), che non ho trovato registrato in nessun dizionario italiano moderno. Un derivato "nuovo", ma comunque prevedibile, è il verbo petessar "bere liquori" (var. di ciuciar e sbevazar), cfr. i già elencati petessante, petesser, e petesson GDDT, che presuppongono, appunto, un *petessar, anche se casualmente non fosse attestato, cfr. P. Sancin "Daghe de bora" (Udine, 1987) p. 95 "la me andava zò a petesar". E si rammenti anche Petesson, soprannome di un pellicciaio triestino (certo Bruno Kufersin, cfr. L. Santin-L. Valentini "Il Meridiano" 1-12-1988). Sono tutte voci che riconfermano - se ce n'era bisogno - la vitalità in ambito dialettale della parola-base petès.

2. Diffusione in area istriana. Ne danno conferma L. Farina VP 3-6-1988 p. 8 (per l'istroveneto in genere), S. A. Stell “Da la fersora ale bronze" (Trieste-Fiume 1990) p. 34 (Pola), B. Mangini AMSIA 90 (1990) p. 282 e F. Ursini LRL III (1989) p. 54 (sempre per Pola). Ad Isola petes era sentito come triestinismo (Vascotto), a Pola,

1 Sul concetto di “caratterizzante” v.M. Doria, L'anima del dialetto triestino. Le parole caratterizzanti, “Il Banco di Lettura” I, 1 (1988) pp. 9-13. 
a Fiume e a Isola risultano attestati anche petesseria e petesson (N. Milani-Kruljac VP 17-8-1989 p. 6 (A. Vascotto cit., N.N. VP 9-1-1988 p.6).

3. Diffusione nello sloveno istriano. Troviamo attestata a Valmovrasa la forma peteš "acquavite" (F. Paclich p. 365)

4. Zona friulana (Gorizia compresa). Accanto a petes e petessarie, citati in GDDT, saranno da aggiungere le forme petez (accanto a petes) "acquavite, liquore alcolico, superalcolico" e petezarie "liquoreria": cosi il Faggin. Si tratta di forme preziose, in quanto corroborano la genuinità della forma bisiacca petez (v. avanti), pure con l'affricata: se ne deduce, quindi, che è petes la forma secondaria rispetto a petez, primario, e non - come si poteva anche sospettare - viceversa. Nel Faggin petes, -ez è tenuto separato da peteč "pettegolezzo", anche "impiccio, imbarazzo", benché sia noto che nel friulano di tipo udinese i due suoni di affricata, $z$ e $\breve{c}$, tendano a confondersi e a sostituirsi l'uno con l'altro. Come a dire che in ultima analisi peteč e petez (-es) potrebbero essere anche corradicali. Infine, importantissima, l'attestazione goriziana peteze (ancora masch.) "acquavite, per favorire la digestione" dell'a. 1628 (R.M. Cossar "Cara vecchia Gorizia", Gorizia 1981, p. 26), unica attestazione della nostra voce risalente a un'epoca sufficientemente "antica" e nuovamente, caratterizzata dall'affricata $-z$ (nonché da una -e epitetica, come nell'attestazione triestina di due secoli più tardi).

5. Zona bisiacca. Vi accenna anche Doria GDDT Suppl. La situazione complessiva a prima vista può sembrare ancora un po' confusa:

I. Esiste un petez sia (a) col significato di "grappa, acquavite" ecc., sia (b) al pl. col significato di "affari intimi, pettegolezzi". Da (a) abbiamo i derivati petezon, pete$z a r$, e petezaria, sempre con l'affricata (naturalmente sorda). Evidentemente nel bisiacco sono espressi con la stessa forma i significati che nel friul. del Faggin risalgono a due voci separate, petes, -ez e peteč.

II. Esiste, accanto a petezar anche un petezar, con affricata sonora, col significato di ital. (s)petezzare. E, distinto dal petezon sopra menzionato, un petezon (sempre con l'affricata sonora) "scoreggione". E' assai probabile che questi petezar e petezon siano tali e quali (o coincidano) con ital. (s)petezzare, caratterizzato anch'esso dall'affricata sonora, siano quindi voci che non hanno nulla a che fare, almeno direttamente, con il nostro petez, petes ed indeboliscano - a mio avviso - l'etimo di quest'ultimo (v. avanti) da peto "flatulenza".

Sfrondata di questa appendice, ripetiamo, la famiglia di parole bisiacche incentrate su petes ricalca abbastanza da vicino la situazione ricostruibile per il friulano.

6. Zona ladina. Risulta in parte già tratteggiata dal Doria, il quale cita il cortin. impetezà "brillo" (e inoltre - e su ciò ritorneremo più avanti - "contagiato da malattia venerea" e anche "indebitato"). La stessa parola è inclusa anche nel nuovo dizionario del Croatto (impetesà), provvisto però, dei soli due primi significati. Il semplice petès risulta però attestato anche nell'auronzese (Zandegiacomo-Lugan) e l'aggettivo (in verità un part. pass.) *impetessè va posto alla base del roccapietorese (gerg.) insepetè 
(nota la metatesi!) "ubriaco fradicio" (Pallabazzer, cfr. A. Zamboni in "Saggi Zolli", Padova 1991, p. 460).

5.1. Etimi già proposti. Ai cinque etimi elencati dal Doria 1. retrogrado da petessaria, fr. pâtisserie "pasticceria-liquoreria", 2. slov. pitje, 3. lat. pitisso, 4. ital. petecchia (Vidossi), 5. da ital. (venez.) suffissato peto "flatulenza" (Doria), ne aggiungo un sesto, franc. pète(-)sec "uomo forte, rigido, autoritario" (il carattere del cliente che frequenta la liquoreria), F. de Farolfi in L. Grassi "Le insegne dell'ospitalità" (Trieste 1985 p. 8), etimo di cui però è fin troppo evidente l'infondatezza; potrebbe, piuttosto, aiutarci nella nostra ricerca un interessante dettaglio riguardante l'immigrazione a Trieste agli inizi del XIX secolo: nel 1839 arriva a Trieste certo Nicolò Pitt "di Zerzivento" (dunque dalla Carnia), cui viene concessa la licenza della vendita di "bibite spiritose" (P. Covre "Il Piccolo" 29.7.1990).

Da questi molteplici dati, presi nel loro insieme (l'esistenza di un petes, -ez in friulano e ladino cadorino, la circostanza che nelle zone bisiacca, friulana e cadorina compaiano varianti con l'affricata anziché con la sibilante, il fatto che nei primi dell'800 immigrano a Trieste liquoristi carnielli) si ricava l'impressione che la voce (e la "cosa") sia, rispetto, a Trieste, di provenienza settentrionale e, contemporaneamente, che le attestazioni in Istria e nel Quarnero (tutte tardive) siano dovute al successivo espandersi del triestino, con le sue voci più caratterizzanti, a S. e a S.E. (fin nello sloveno istriano) della città adriatica. Un movimento inverso (da Trieste, o, addirittura, da Pola o Fiume) verso il Friuli e il Cadore, cui sembra dar credito - se ho ben capito - lo Zamboni (cit.), pare qui, effettivamente, da escludersi, qualora si tenga ben presente la funzione particolare, quasi di cerniera, del dialetto triestino, che accoglie e smista verso S. termini lessicali provenienti da N., in virtù della sua posizione geografica e del prestigio che esso godette in ambito giuliano, verso la metà dell' 800 , all'epoca delle massime fortune emporiali della città ${ }^{2}$.

5.2. Nuove proposte etimologiche. Se è stato relativamente facile ricostruire la strada per la quale la parola, già carica del suo significato specifico, è giunta dal N. o N.O. a Trieste per proseguire, poi, sorta di Wanderwort, verso S. e S.E., non altrettanto agevole è lo stabilirne l'etimologia; ossia giustificare il suo particolare assetto, sia dal lato del suo signifiant che del suo signifié nella lingua o gruppo di lingue d'origine. Qui veramente troviamo interrotti quei "ponti" che tanto ci servirebbero per fissare la parola all'interno di un ambito particolare, ben preciso e concreto, ambito che a priori non è possibile, appunto, ipotizzare dato l'intrecciarsi, in coteste lingue e dialetti, che possiamo ben definire come "alpini" o meglio "prealpini", di vari filoni definibili via via come indigeni (locali), veneti, tedeschi, anche slavi, ognuno dei quali può essersi congelato in una forma e/o significato del tutto particolari e irripetibili. In altri termini,

2 Un altro chiaro esempio, proprio all'interno della stessa sfera nozionistica, di parola che "scende" da N. verso S. è il triestino trapa "acquavite di vinacce". Tratta da ted. Treber "vinacce" mantiene intatto questo suo significato solo nel friul. (trape) e nel bisiacco, mentre già in gradese, oltre che "vinacce" significa anche "acquavite (di vinacce)" e in triest. possiede solo questo secondo significato (che è anche l'unico attestato per questa voce in Istria) (cfr. GDDT e GDDT Suppl.). 
non possiamo procedere ulteriormente se non per tentativi. Ora si dà il caso che è giusto affermare che petes o petez è parola cadorina (o carniella), ma quivi, ci domandiamo, che cosa essa veramente rappresenta? Essa aveva sempre questa forma e questo significato (/-i) e quest'ultimo si concilia, a parte che la distillazione delle vinacce è pratica non certo antichissima, con tutta una serie di Realien cronologicamente fissabili?

Tre, a mio modo di vedere, sono le vie che si possono battere in vista di un etimo accettabile. Le tratterò qui in modo molto schematico, rimandando ad altra occasione il compito di studiarne i singoli particolari.

1) La voce è connessa con friul. peta "focaccia" (da PITTA, REW 6546), di cui sarebbe una specie di diminutivo-peggiorativo, il quale avrebbe assunto il significato di "protuberanza cutanea appiattita", "pustoletta", poi "eritema", "la faccia paonazza dell'ubriaco", l'ubriachezza stessa. Gli inizi di questo sviluppo semantico sarebbero riscontrabili in altre voci neolatine facenti capo allo stesso PĬTTA "Kuchen", precisamente rum. "pată", "Fleck, Flicken" e portogh. peta "Fleck im Auge des Pferdes". Siamo vicini, come si vede, per la parte semantica, all'etimo del Vidossi, anche se egli vi era arrivato per altre vie. L'etimo, comunque, si semplificherebbe qualora a base della voce rumena (e portogh.) e di petez si postulasse anziché PǏTTA la base PICTĀRE "dipingere" (cosí il Cioranescu s.v. per la voce rumena, nonostante alcune difficoltà d'ordine fonetico). Come a dire che il ricostruito cador. o friul. peta "pustola rossastra" o, sim. manterrebbe l'accezione coloristica insita nell'etimo PICTĀRE. Tutto ciò a condizione che friul. peta "focaccia" fosse una parola d'etimo completamente diverso e solo casualmente omofona alla base di partenza da noi ipotizzata. $\mathrm{Ci}$ sorreggerebbe in questa nostra etimologia l'esistenza nel rum. stesso di un dimin. petiţa sf. "mancha pequeña" ecc. e anche in ambito veneto il chiogg. petecia "macchia rossa, scalmana" nonché petassier "che ha la faccia paonazza" (non necessariamente italianismi tratti da petecchia).

2) La voce è connessa con ital. petecchia, quindi tratta, come questa, da lat. (IM)PETİCULA (DEI, DELI), variante di IMPITİGINEM, da cui triest. pe- e pidin, istr. (pir.) lipidin, friul. pedign (per altre forme rimandiamo al REW cit.). Per accettare tale etimo sarebbe, però, necessario, causa il $t$ intervocalico conservato, ritenere la parola un prestito dalla lingua letteraria. Resta, naturalmente, anche da spiegare, parallelamente a quanto supposto per l'etimo precedente, il passaggio semantico da "impetigine" o "pustoletta" ad "eritema", "rossore dell'ubriacone" ecc. Ovviamente con questo etimo petes non avrebbe nessun legame con il quasi omofono friul. peteč e nemmeno con la serie di venez. pettegolo e affini (e, di riflesso, con veneto petar).

3) La voce, in questo caso un tutt'uno con friul. peteč "chiacchiera" e anche "imbroglio", apparterrebbe alla vasta famiglia (radicale pet $(t)-$ ), cui risalirebbero alcune voci veneziane (e poi ital.) pettegolo (il cui etimo, però, nonostante il DELI non è affatto chiaro) ${ }^{3}$. Nel caso si intenda stabilime l'etimo nell'ital. (o/e venez.) peto

3 Fra i vari significati di ven. (anche friul. e bis.) petez(o) e sim. da scartare, ai fini della nostra ricerca, 
"flatumenza" (lat. PEDĬTĀRE), ricordiamo che questo ha contro di sé il fatto che l'ital. (e il veneto) hanno un pettezzare e petezzo (con l'affricata sonora), quindi non raccordabile in nessun modo ai nostri petes o petez e derivati (v. avanti), a meno che non si ammetta, antieconomicamente, una pluralità di suffissi per giustificare, separatamente, le due serie di derivati. Qui i passaggi semantici sono ancora più numerosi $\mathrm{e}$ complessi, anche se è facile un' evoluzione da "imbarazzo" o "imbroglio" a "indebitamento" o "malattia venerea" (in ambedue i casi chi ne è colpito si trova "nei guai"). Le implicazioni, per un controllo dei Realien, sono numerose, né si dimentichi che il radicale pet $(t)$ - potrebbe essere lo stesso di veneziano petar "affibbiare" ecc., per il quale non si esclude nemmeno un'origine onomatopeica (v.C. Marcato RELV p. 115) o un ricorso, in extremis, al PICTĀRE di cui si è già discusso.

Concludendo, non ho voluto far altro, in questa sede, che illustrare sommariamente alcune proposte etimologiche che mi son sembrate a un primo momento, abbastanza sensate. Ovviamente, in previsione di una scelta finale, ognuna di queste ipotesi richiederebbe un trattamento più approfondito, tenendo nel debito conto tutti $i$ valori possibili assunti da ciascun termine delle famiglie lessicali elencate, sia in ambito veneto, che friulano "ladino"; tanto più che, con tale approfondimento, potrebbe capitarci sotto mano qualche attestazione-chiave, tale da rischiarare tutta la documentazione in nostro possesso e condurci verso la sospirata soluzione. Ma non osando sperare che ciò possa arrivare tanto presto, accontentiamoci di aver tratteggiato le vie essenziali della ricerca e aver risolto efficacemente il problema della "storia" più recente del nostro petes.

\section{ABBREVIAZIONI BIBLIOGRAFICHE}

AMSIA "Atti e memorie della Società Istriana di Archeologia e di Storia Patria", Parenzo (poi Trieste).

Cioranescu A. Cioranescu, Diccionario etimológico rumano, La Laguna/Tenerife 1958-1966.

GDDT (Suppl.) M. Doria, Grande dizionario del dialetto triestino, Trieste 1987.

LRL "Lexikon der romanistischen Linguistik", Tübingen 1989.

DEII C. Battisti-G. Alessio, Dizionario etimologico italiano, Firenze 1950-1957.

DELI M. Cortelazzo - P. Zolli, Dizionario etimologico della lingua italiana, Bologna 1979-1988.

Marcato RELV C. Marcato, Ricerche etimologiche sul lessico veneto, Padova 1982. 
Paclich F. Paclich, Slovenski dialektološki leksikalni atlas Koprske Pokrajine SDLA-KP (II), Tesi Scuola Super. Interpreti e traduttori, Univ. Trieste, a.a. 1989-1990.

REW W. Meyer-Lübke, Romanisches etymologisches Wörterbuch, Heidelberg 1935.

Saggi Zolli "Saggi di linguistica e di letteratura in memoria di Paolo Zolli", Padova 1991.

Vascotto A. Vascotto, Voci della parlata isolana nella prima metà di questo secolo, Imola 1987.

VP "La voce del Popolo", Fiume (quotidiano).

Povzetek

ZGODOVINA IN ETIMOLOGIJA TRŽAŠKIH IZRAZOV

Prispevek daje zgodovinski pregled rabe izraza petès ‘žganje, močna alkoholna pijača slabe kakovosti' v zadnjih 150 letih. Izhaja sicer iz Dorijevega narečnega slovarja, ponuja pa nekaj novih etimologij, obenem pa opozarja na možnost onomatopeične razlage. Izraz ni samo tržaški; pozna ga vsa Istra in del Furlanije. 